\title{
The Trapezoidal Rule for Computing Cauchy Principal Value Integral on Circle
}

\author{
Jin $\operatorname{Li}^{1,2}$ \\ ${ }^{1}$ School of Science, Shandong Jianzhu University, Jinan 250101, China \\ ${ }^{2}$ School of Mathematics, Shandong University, Jinan 250100, China \\ Correspondence should be addressed to Jin Li; lijin@lsec.cc.ac.cn
}

Received 27 July 2015; Accepted 20 September 2015

Academic Editor: Kishin Sadarangani

Copyright ( 92015 Jin Li. This is an open access article distributed under the Creative Commons Attribution License, which permits unrestricted use, distribution, and reproduction in any medium, provided the original work is properly cited.

\begin{abstract}
The composite trapezoidal rule for the computation of Cauchy principal value integral with the singular $\operatorname{kernel} \cot ((x-s) / 2)$ is discussed. Our study is based on the investigation of the pointwise superconvergence phenomenon; that is, when the singular point coincides with some a priori known point, the convergence rate of the trapezoidal rule is higher than what is globally possible. We show that the superconvergence rate of the composite trapezoidal rule occurs at middle of each subinterval and obtain the corresponding superconvergence error estimate. Some numerical examples are provided to validate the theoretical analysis.
\end{abstract}

\section{Introduction}

Consider the Cauchy principal integral

$$
\begin{aligned}
I(f ; s)=f_{c}^{c+2 \pi} \cot \frac{x-s}{2} f(x) d x=g(s), & \\
& \\
& s \in(0,2 \pi),
\end{aligned}
$$

where $f_{c}^{c+2 \pi}$ denotes a Cauchy principal value integral and $s$ is the singular point.

There are several different definitions which can be proved equally, such as the definition of subtraction of the singularity, regularity definition, and direct definition.

In this paper we adopt the following one:

$$
\begin{aligned}
& f_{c}^{c+2 \pi} \cot \frac{x-s}{2} f(x) d x \\
& \quad=\lim _{\varepsilon \rightarrow 0}\left\{\int_{0}^{s-\varepsilon} \cot \frac{x-s}{2} f(x) d x\right. \\
&\left.\quad+\int_{s+\varepsilon}^{2 \pi} \cot \frac{x-s}{2} f(x) d x\right\} .
\end{aligned}
$$

Cauchy principal value integral has recently attracted a lot of attention; see, for example, [1]. The main reason for this interest is probably due to the fact that integral equations with Cauchy principal value integrals have been shown to be an adequate tool [1] for the modeling of many physical situations, such as acoustics, fluid mechanics, elasticity, fracture mechanics, and electromagnetic scattering problems. Numerous work has been devoted in developing efficient quadrature formulas, such as the Gaussian method [2-8], the Newton-Cotes methods [9-13], spline methods [14, 15], and some other methods [16-22].

It is the aim of this paper to investigate the superconvergence phenomenon of trapezoidal rule and, in particular, to derive error estimates. In this paper the density function $f(x)$ is replaced by the approximation function $f_{L}(x)$ while the singular kernel $\cot ((x-s) / 2)$ is computed $\cot \left(\left(x_{i}-s\right) / 2\right)$ in each subinterval, where $f_{L}(x)$ is the trapezoidal rule. This method is different from the semidiscrete methods and the order of singularity kernel can be reduced somehow which was firstly presented by Linz in the paper to calculate the hypersingular integral on interval. He used the trapezoidal rule and Simpson rule to approximate the density function $f(x)$ and the convergence rate was $O\left(h^{k}\right), k=1,2$, when the singular point is always located at the middle of certain subinterval. 
The superconvergence of composite Newton-Cotes rules for Hadamard finite-part integrals was studied in [23, 24], where the superconvergence rate and the superconvergence point were presented, respectively. In $[25,26]$ the classical composite midpoint rectangle rule and classical composite trapezoidal rectangle rule for the computation of Cauchy principal value integrals are discussed. When the singular point coincides with some a priori known point, the convergence rate of the midpoint rectangle rule is higher than the global one, the same as the Riemann integral, which is called superconvergence phenomenon.

This paper focuses on the superconvergence of trapezoidal rule for Cauchy principle integrals in a circle. Based on the investigation of the pointwise superconvergence phenomenon, that is, when the singular point coincides with some a priori known point, the convergence rate of the trapezoidal rule is higher than what is globally possible. We show that the superconvergence rate of the composite trapezoidal rule occurs at middle of each subinterval and obtain the corresponding superconvergence error estimate. We prove both theoretically and numerically that the composite trapezoidal rule reaches the superconvergence rate $O\left(h^{2}\right)$ when the local coordinate of the singular point $s$ is 0 .

The rest of this paper is organized as follows. In Section 2, after introducing some basic formulas of the rectangle rule, we present the main results. In Section 3 we perform the proof. Finally, several numerical examples are provided to validate our analysis.

\section{Main Result}

Let $c=x_{0}<x_{1}<\cdots<x_{n-1}<x_{n}=c+2 \pi$ be a uniform partition of the interval $[c, c+2 \pi]$ with mesh size $h=2 \pi / n$. Define by $f_{L}(x)$ the piecewise line interpolant for $f(x)$ :

$$
f_{L}(x)=\frac{f\left(x_{i}\right)}{2}, \quad i=0,1, \ldots, n,
$$

and a linear transformation

$$
x=\widehat{x}_{i}(\tau):=\frac{(\tau+1)\left(x_{i+1}-x_{i}\right)}{2}+x_{i}, \quad \tau \in[-1,1],
$$

from the reference element $[-1,1]$ to the subinterval $\left[x_{i}, x_{i+1}\right]$. Replacing $f(x)$ in $(2)$ with $f_{L}(x)$ gives the composite trapezoidal rule:

$$
I_{n}(f ; s):=\sum_{i=0}^{n} \omega_{i}(s) f\left(x_{i}\right)=I(f ; s)-E_{n}(f),
$$

where $\omega_{i}(s)$ denote the Cotes coefficients given by

$$
\omega_{i}(s)=\frac{h}{2} \cot \frac{x_{i}-s}{2} .
$$

We also define

$$
k_{s}(x)= \begin{cases}(x-s) \cot \frac{x-s}{2}, & x \neq s, \\ 2, & x=s .\end{cases}
$$

Theorem 1. Assume $f(x) \in C^{1}[a, b]$. For the trapezoidal rule $I_{n}(f, s)$ is defined as (5). Assuming that $s=x_{m}+(1+\tau) h / 2$, there exists a positive constant $C$, independent of $h$ and $s$, such that

$$
\left|E_{n}(f)\right| \leq C \max \left\{\left|k_{s}(x)\right|\right\}(|\ln h|+|\ln \gamma(\tau)|) h,
$$

where

$$
\gamma(\tau)=\min _{0 \leq i \leq n} \frac{\left|s-x_{i}\right|}{h}=\frac{1-|\tau|}{2} .
$$

Proof. Let $R(x)=f(x)-f\left(x_{i}\right) / 2-f\left(x_{i+1}\right) / 2$; then we have $|R(x)| \leq C h$. Thus

$$
\begin{aligned}
& E_{n}(f)=\sum_{i=0}^{n} \int_{x_{i}}^{x_{i+1}}\left[\cot \frac{x-s}{2} f(x)-\frac{f\left(x_{i}\right)}{2} \cot \frac{x_{i}-s}{2}\right. \\
& \left.\quad-\frac{f\left(x_{i+1}\right)}{2} \cot \frac{x_{i+1}-s}{2}\right] d x=\sum_{i=0}^{n} f_{x_{i}}^{x_{i+1}} \frac{R(x)}{x-s} d x \\
& +\sum_{i=0}^{n} \int_{x_{i}}^{x_{i+1}} \frac{k_{s}(x)-2}{x-s} R(x) d x \\
& +\sum_{i=0}^{n} \int_{x_{i}}^{x_{i+1}}\left[\cot \frac{x-s}{2}-\cot \frac{x_{i}-s}{2}\right] \frac{f\left(x_{i}\right)}{2} d x \\
& +\sum_{i=0}^{n} \int_{x_{i}}^{x_{i+1}}\left[\cot \frac{x-s}{2} \cot \frac{x_{i+1}-s}{2}\right] \frac{f\left(x_{i+1}\right)}{2} d x .
\end{aligned}
$$

For the first part of (10), we have

$$
\begin{aligned}
& \left|\sum_{i=0, i \neq m}^{n-1} \int_{x_{i}}^{x_{i+1}} \frac{R(x)}{x-s} d x\right| \leq C \max \left\{\left|k_{s}(x)\right|\right\} h \\
& \cdot\left(\int_{c}^{x_{m}} \frac{1}{s-x} d x+\int_{x_{m+1}}^{c+2 \pi} \frac{1}{x-s} d x\right)=C \\
& \cdot \max \left\{\left|k_{s}(x)\right|\right\} h \ln \frac{(c+2 \pi-s)(s-c)}{\left(x_{m+1}-s\right)\left(s-x_{m}\right)} \leq C \\
& \cdot \max \left\{\left|k_{s}(x)\right|\right\}(|\ln h|+|\ln \gamma(\tau)|) h .
\end{aligned}
$$

For the second part of (10), we have

$$
\begin{aligned}
& \left|\int_{x_{m}}^{x_{m+1}} \frac{R(x)}{x-s} d x\right| \\
& \quad \leq\left|\int_{x_{m}}^{x_{m+1}} \frac{R(x)-R(s)}{x-s} d x\right|+\left|R(s) \ln \frac{x_{m+1}-s}{s-x_{m}}\right| \\
& \quad \leq C h|\ln \gamma(\tau)|, \\
& \left|f_{c}^{c+2 \pi} \frac{k_{s}(x)-2}{x-s} R(x) d x\right| \leq C h\left|\int_{c}^{c+2 \pi} \frac{k_{s}(x)-2}{x-s} d x\right| \\
& \quad \leq C h|\ln \gamma(\tau)| .
\end{aligned}
$$


For the third part of (10), we have

$$
\begin{aligned}
& \left|\sum_{i=0}^{n} f_{x_{i}}^{x_{i+1}}\left[\cot \frac{x-s}{2}-\cot \frac{x_{i}-s}{2}\right] \frac{f\left(x_{i}\right)}{2} d x\right| \\
& \leq\left|\sum_{i=0, i \neq m}^{n} \int_{x_{i}}^{x_{i+1}}\left[\cot \frac{x-s}{2}-\cot \frac{x_{i}-s}{2}\right] \frac{f\left(x_{i}\right)}{2} d x\right| \quad \begin{array}{c}
\text { Combining (11), (13), and (14) to }\left\{\left|k_{s}(x)\right|\right\}(|\ln h|+ \\
\text { pleted. }
\end{array} \\
& +\left|\int_{x_{m}}^{x_{m+1}}\left[\cot \frac{x-s}{2}-\cot \frac{x_{i}-s}{2}\right] \frac{f\left(x_{i}\right)}{2} d x\right| \\
& I_{n, i}(s)=\left\{\begin{array}{l}
\int_{x_{m}}^{x_{m+1}}\left[\cot \frac{x-s}{2}-\frac{1}{2} \cot \frac{x_{i}-s}{2}-\frac{1}{2} \cot \frac{x_{i+1}-s}{2}\right] d x, \quad i=m, \\
\int_{x_{i}}^{x_{i+1}}\left[\cot \frac{x-s}{2}-\frac{1}{2} \cot \frac{x_{i}-s}{2}-\frac{1}{2} \cot \frac{x_{i+1}-s}{2}\right] d x, \quad i \neq m .
\end{array}\right.
\end{aligned}
$$

$$
\begin{aligned}
& =C \max \left\{\left|k_{s}(x)\right|\right\} h \ln \frac{(c+2 \pi-s)(s-c)}{\left(x_{m+1}-s\right)\left(s-x_{m}\right)} \\
& \leq C \max \left\{\left|k_{s}(x)\right|\right\}(|\ln h|+|\ln \gamma(\tau)|) h .
\end{aligned}
$$

Combining (11), (13), and (14) together, the proof is com-
Lemma 2. Assume $s=x_{m}+(\tau+1) h / 2$ with $\tau \in(-1,1)$. Let $I_{n, i}(s)$ be defined by (15); then there holds that

$$
\begin{aligned}
I_{n, i}(s)= & -2 \sum_{k=1}^{\infty} \frac{1}{k}\left[\cos k\left(x_{i+1}-s\right)-\cos k\left(x_{i}-s\right)\right] \\
& +h \sum_{k=1}^{\infty}\left[\sin k\left(x_{i+1}-s\right)+\sin k\left(x_{i}-s\right)\right] .
\end{aligned}
$$

Proof. For $i=m$, by the definition of Cauchy principal value integral, we have

$$
\begin{aligned}
I_{n, m}(s) & =\lim _{\varepsilon \rightarrow 0}\left(\int_{x_{m}}^{s-\varepsilon}+\int_{s+\varepsilon}^{x_{m+1}}\right) \\
\cdot & {\left[\cot \frac{x-s}{2}-\frac{1}{2} \cot \frac{x_{m}-s}{2}-\frac{1}{2} \cot \frac{x_{m+1}-s}{2}\right] d x } \\
= & 2 \ln \left(2 \sin \frac{x_{m}-s}{2}\right)-2 \ln \left(2 \sin \frac{x_{m+1}-s}{2}\right) \\
+ & h\left[-\frac{1}{2} \cot \frac{x_{m}-s}{2}-\frac{1}{2} \cot \frac{x_{m+1}-s}{2}\right] .
\end{aligned}
$$

For $i \neq m$, taking integration by parts on the correspondent Riemann integral, we have

$$
\begin{aligned}
I_{n, i}(s)= & 2 \ln \left(2 \sin \frac{x_{i}-s}{2}\right)-2 \ln \left(2 \sin \frac{x_{i+1}-s}{2}\right) \\
& +h\left[-\frac{1}{2} \cot \frac{x_{i}-s}{2}-\frac{1}{2} \cot \frac{x_{i+1}-s}{2}\right] .
\end{aligned}
$$

Now, by using the well-known identity,

$$
\begin{aligned}
\ln \left|2 \sin \frac{x}{2}\right| & =-\sum_{n=1}^{\infty} \frac{1}{n} \cos n x, \\
\frac{1}{2} \cot \frac{x}{2} & =\sum_{n=1}^{\infty} \sin n x .
\end{aligned}
$$

The proof is completed.
Lemma 3. Under the same assumptions of Lemma 2, there holds that

$$
\sum_{i=1}^{n} I_{n, i}(s)=-2 \pi \tan \frac{\tau \pi}{2} .
$$

Proof. By (15), we have

$$
\begin{aligned}
\sum_{i=1}^{n} I_{n, i}(s)= & -2 \sum_{k=1}^{\infty} \frac{1}{k}\left(\cos k\left(x_{i+1}-s\right)-\cos k\left(x_{i}-s\right)\right) \\
& +h \sum_{k=1}^{\infty}\left(\sin k\left(x_{i+1}-s\right)+\sin k\left(x_{i}-s\right)\right) \\
= & 2 h \sum_{k=1}^{\infty} \sum_{i=1}^{n} \sin \left[k\left(x_{i}-s\right)\right] \\
= & 2 h \sum_{j=1}^{\infty} n \sin \left[n j\left(x_{1}-s\right)\right] \\
= & 4 \pi \sum_{j=1}^{\infty} \sin [j(1+\tau) \pi]=2 \pi \cot \frac{(1+\tau) \pi}{2} \\
= & -2 \pi \tan \frac{\tau \pi}{2} .
\end{aligned}
$$

The proof is completed.

Theorem 4. Assume $f(x) \in C^{2}[a, b]$. For the trapezoidal rule $I_{n}(f, s)$ is defined as (5). Assuming that $s=x_{m}+(1+\tau) h / 2$, there exists a positive constant $C$, independent of $h$ and $s$, such that

$$
E_{n}(f)=-2 f(s) \pi \tan \frac{\tau \pi}{2}+\mathscr{R}_{n}(s),
$$

where

$$
\left|\mathscr{R}_{n}(s)\right| \leq C \max \left\{\left|k_{s}(x)\right|\right\}(|\ln h|+|\ln \gamma(\tau)|) h^{2} .
$$

$\gamma(\tau)$ is defined as (9). 
It is known that the global convergence rate of the composite trapezoidal rule is lower than Riemann integral.

\section{Proof of the Theorem}

In this section, we study the superconvergence of the composite trapezoidal rule for Cauchy principle integrals.

3.1. Preliminaries. In the following analysis, $C$ will denote a generic constant that is independent of $h$ and $s$ and it may have different values in different places.

Lemma 5. Under the same assumptions of Theorem 4, it holds that

$$
\begin{aligned}
& \cot \frac{x-s}{2} f(x)-\frac{f\left(x_{i}\right)}{2} \cot \frac{x_{i}-s}{2}-\frac{f\left(x_{i+1}\right)}{2} \\
& \cdot \cot \frac{x_{i+1}-s}{2}=\left[\cot \frac{x-s}{2}-\frac{1}{2} \cot \frac{x_{i}-s}{2}\right. \\
& \left.-\frac{1}{2} \cot \frac{x_{i+1}-s}{2}\right] f(s)+\left[(x-s) \cot \frac{x-s}{2}\right. \\
& \left.-\frac{x_{i}-s}{2} \cot \frac{x_{i}-s}{2}-\frac{x_{i+1}-s}{2} \cot \frac{x_{i+1}-s}{2}\right] f^{\prime}(s) \\
& +R_{f}^{1}(x)+R_{f}^{2}(x)+R_{f}^{3}(x)+R_{f}^{4}(x),
\end{aligned}
$$

where

$$
\begin{aligned}
& R_{f}^{1}(x)=\left[\cot \frac{x-s}{2}-\frac{1}{2} \cot \frac{x_{i}-s}{2}-\frac{1}{2} \cot \frac{x_{i+1}-s}{2}\right] \\
& \cdot \frac{1}{2} f^{\prime \prime}\left(\beta_{i 1}\right)(x-s)^{2}, \\
& R_{f}^{2}(x)=-\frac{f^{\prime \prime}\left(\alpha_{i 1}\right)}{2}\left(x_{i}-x\right)^{2} \cot \frac{x_{i}-s}{2}, \\
& R_{f}^{3}(x)=-\frac{f^{\prime \prime}\left(\alpha_{i 2}\right)}{2}\left(x_{i+1}-x\right)^{2} \cot \frac{x_{i+1}-s}{2}, \\
& R_{f}^{4}(x)=-\frac{1}{2}\left[\left(x_{i}-x\right) \cot \frac{x_{i}-s}{2}\right. \\
& \left.+\left(x_{i+1}-x\right) \cot \frac{x_{i+1}-s}{2}\right] f^{\prime \prime}\left(\beta_{i 2}\right)(x-s) .
\end{aligned}
$$

Proof. Performing Taylor expansion of $f_{L}(x)$ at the point $x$, we have

$$
\begin{aligned}
f\left(x_{i}\right)= & f(x)+f^{\prime}(x)\left(x_{i}-x\right) \\
& +\frac{f^{\prime \prime}\left(\alpha_{i 1}\right)}{2}\left(x_{i}-x\right)^{2}, \\
f\left(x_{i+1}\right)= & f(x)+f^{\prime}(x)\left(x_{i+1}-x\right) \\
& +\frac{f^{\prime \prime}\left(\alpha_{i 2}\right)}{2}\left(x_{i+1}-x\right)^{2} .
\end{aligned}
$$

Similarly, we have

$$
\begin{aligned}
& f(x)=f(s)+f^{\prime}(s)(x-s)+\frac{1}{2} f^{\prime \prime}\left(\beta_{i 1}\right)(x-s)^{2}, \\
& f^{\prime}(x)=f^{\prime}(s)+f^{\prime \prime}\left(\beta_{i 2}\right)(x-s) .
\end{aligned}
$$

We set

$$
\begin{aligned}
& E_{m}(x)=\cot \frac{x-s}{2} f(x)-\frac{f\left(x_{m}\right)}{2} \cot \frac{x_{m}-s}{2} \\
& -\frac{f\left(x_{m+1}\right)}{2} \cot \frac{x_{m+1}-s}{2}-\left[\cot \frac{x-s}{2}\right. \\
& \left.-\frac{1}{2} \cot \frac{x_{m}-s}{2}-\frac{1}{2} \cot \frac{x_{m+1}-s}{2}\right] f(s) \\
& +\left[(x-s) \cot \frac{x-s}{2}-\frac{x_{m}-s}{2} \cot \frac{x_{m}-s}{2}\right. \\
& \left.-\frac{x_{m+1}-s}{2} \cot \frac{x_{m+1}-s}{2}\right] f^{\prime}(s) .
\end{aligned}
$$

Lemma 6. Let $f(x) \in C^{2}[a, b]$, denote $E_{m}(x)$ to be the error functional for the composite trapezoidal rule, and assume $s \neq$ $x_{i}$ for any $i=0,1,2, \ldots, n$; then there holds

$$
\left|f_{x_{m}}^{x_{m+1}} \cot \frac{x-s}{2} E_{m}(x) d x\right| \leq C h^{2}|\ln \gamma(\tau)| .
$$

Proof. As $f(x) \in C^{2}[a, b]$, we get $E_{i}(x) \in C^{2}[a, b]$. We have

$$
\begin{aligned}
& \int_{x_{m}}^{x_{m+1}} \cot \frac{x-s}{2} E_{m}(x) d x \\
& =2 f_{x_{m}}^{x_{m+1}} \frac{E_{m}(x)}{x-s} d x+\int_{x_{m}}^{x_{m+1}} \frac{k_{s}(x)-2}{x-s} E_{m}(x) d x .
\end{aligned}
$$

For the first part of (30), by the definition of

$$
\int_{a}^{b} \frac{f(x)}{x-s} d x=\int_{a}^{b} \frac{f(x)-f(s)}{x-s} d x+f(s) \ln \left|\frac{b-s}{s-a}\right|,
$$

then we have

$$
\begin{aligned}
\int_{x_{m}}^{x_{m+1}} \frac{E_{m}(x)}{x-s} d x= & \int_{x_{m}}^{x_{m+1}} \frac{E_{m}(x)-E_{m}(s)}{x-s} d x \\
& +E_{m}(s) \ln \frac{x_{m+1}-s}{s-x_{m}} ;
\end{aligned}
$$

then we get

$$
\begin{aligned}
\left|\int_{x_{m}}^{x_{m+1}} \frac{E_{m}(x)}{x-s} d x\right| \leq & \left|\int_{x_{m}}^{x_{m+1}} \frac{E_{m}(x)-E_{m}(s)}{x-s} d x\right| \\
& +\left|E_{m}(s) \ln \frac{x_{m+1}-s}{s-x_{m}}\right| \\
\leq & C h^{2}|\ln \gamma(\tau)| .
\end{aligned}
$$


For the second part of (30), we have

$$
\begin{aligned}
& \left|f_{x_{m}}^{x_{m+1}} \frac{k_{s}(x)-2}{x-s} R(x) d x\right| \leq C h^{2}\left|f_{x_{m}}^{x_{m+1}} \frac{k_{s}(x)-2}{x-s} d x\right| \\
& \quad=C h^{2}\left|f_{x_{m}}^{x_{m+1}} \cot \frac{x-s}{2} d x-\int_{x_{m}}^{x_{m+1}} \frac{2}{x-s} d x\right| \\
& \quad \leq C h^{2}|\ln \gamma(\tau)| .
\end{aligned}
$$

And the proof is completed.

Proof of Theorem 4. By Lemma 5, we have

$$
\begin{aligned}
& \left(\int_{c}^{x_{m}}+\int_{x_{m+1}}^{c+2 \pi}\right)\left[\cot \frac{x-s}{2} f(x)-\frac{f\left(x_{i}\right)}{2} \cot \frac{x_{i}-s}{2}\right. \\
& \left.-\frac{f\left(x_{i+1}\right)}{2} \cot \frac{x_{i+1}-s}{2}\right] d x \\
& =\sum_{i=0, i \neq m}^{n-1} \int_{x_{i}}^{x_{i+1}}\left[\cot \frac{x-s}{2} f(x)-\frac{f\left(x_{i}\right)}{2} \cot \frac{x_{i}-s}{2}\right. \\
& \left.-\frac{f\left(x_{i+1}\right)}{2} \cot \frac{x_{i+1}-s}{2}\right] d x=f(s) \\
& \cdot \sum_{i=0, i \neq m}^{n-1} \int_{x_{i}}^{x_{i+1}}\left[\cot \frac{x-s}{2} f(x)-\frac{1}{2} \cot \frac{x_{i}-s}{2}-\frac{1}{2}\right. \\
& \left.\cdot \cot \frac{x_{i+1}-s}{2}\right] d x+f^{\prime}(s) \sum_{i=0, i \neq m}^{n-1} \int_{x_{i}}^{x_{i+1}}[(x-s) \\
& \cdot \cot \frac{x-s}{2}-\frac{x_{i}-s}{2} \cot \frac{x_{i}-s}{2}-\frac{x_{i+1}-s}{2} \\
& \left.\cdot \cot \frac{x_{i+1}-s}{2}\right] d x+\sum_{i=0, i \neq m}^{n-1} \int_{x_{i}}^{x_{i+1}}\left[\cot \frac{x-s}{2}-\frac{1}{2}\right. \\
& \left.\cdot \cot \frac{x_{i}-s}{2}-\frac{1}{2} \cot \frac{x_{i+1}-s}{2}\right] \frac{1}{2} f^{\prime \prime}\left(\beta_{i 1}\right)(x-s)^{2} d x \\
& -\sum_{i=0, i \neq m}^{n-1} \int_{x_{i}}^{x_{i+1}} \frac{f^{\prime \prime}\left(\alpha_{i 1}\right)}{2}\left(x_{i}-x\right)^{2} \cot \frac{x_{i}-s}{2} d x \\
& -\sum_{i=0, i \neq m}^{n-1} \int_{x_{i}}^{x_{i+1}} \frac{f^{\prime \prime}\left(\alpha_{i 2}\right)}{2}\left(x_{i+1}-x\right)^{2} \cot \frac{x_{i+1}-s}{2} d x
\end{aligned}
$$

$$
\begin{aligned}
& -\sum_{i=0, i \neq m}^{n-1} \int_{x_{i}}^{x_{i+1}} \frac{f^{\prime \prime}\left(\beta_{i 2}\right)(x-s)}{2}\left[\left(x_{i}-x\right) \cot \frac{x_{i}-s}{2}\right. \\
& \left.+\left(x_{i+1}-x\right) \cot \frac{x_{i+1}-s}{2}\right] d x
\end{aligned}
$$

By the definition of $E_{m}(x)$, we have

$$
\begin{aligned}
& f_{x_{m}}^{x_{m+1}}\left[\cot \frac{x-s}{2} f(x)-\frac{f\left(x_{m}\right)}{2} \cot \frac{x_{m}-s}{2}\right. \\
& \left.-\frac{f\left(x_{m+1}\right)}{2} \cot \frac{x_{m+1}-s}{2}\right] d x=\int_{x_{m}}^{x_{m+1}} E_{m}(x) \\
& \cdot \cot \frac{x-s}{2} d x+f(s) f_{x_{m}}^{x_{m+1}}\left[\cot \frac{x-s}{2}\right. \\
& \left.-\frac{1}{2} \cot \frac{x_{m}-s}{2}-\frac{1}{2} \cot \frac{x_{m+1}-s}{2}\right] d x+f^{\prime}(s) \\
& \cdot \int_{x_{m}}^{x_{m+1}}\left[(x-s) \cot \frac{x-s}{2}-\frac{x_{m}-s}{2} \cot \frac{x_{m}-s}{2}\right. \\
& \left.-\frac{x_{m+1}-s}{2} \cot \frac{x_{m+1}-s}{2}\right] d x .
\end{aligned}
$$

Putting (35), (36) together yields

$$
\begin{aligned}
& f_{c}^{c+2 \pi}\left[\cot \frac{x-s}{2} f(x)-\frac{f\left(x_{i}\right)}{2} \cot \frac{x_{i}-s}{2}-\frac{f\left(x_{i+1}\right)}{2}\right. \\
& \left.\cdot \cot \frac{x_{i+1}-s}{2}\right] d x=\sum_{i=0, i \neq m}^{n-1} \int_{x_{i}}^{x_{i+1}}\left[\cot \frac{x-s}{2} f(x)\right. \\
& \left.-\frac{f\left(x_{i}\right)}{2} \cot \frac{x_{i}-s}{2}-\frac{f\left(x_{i+1}\right)}{2} \cot \frac{x_{i+1}-s}{2}\right] d x \\
& +f_{x_{m}}^{x_{m+1}}\left[\cot \frac{x-s}{2} f(x)-\frac{f\left(x_{m}\right)}{2} \cot \frac{x_{m}-s}{2}\right. \\
& \left.-\frac{f\left(x_{m+1}\right)}{2} \cot \frac{x_{m+1}-s}{2}\right] d x=f(s) \\
& \left.\quad-\frac{1}{2} \cot \frac{x_{i+1}-s}{2}\right] d x+f^{\prime}(s) \\
& \quad \sum_{i=0}^{n-1} f_{x_{i}}^{x_{i+1}}\left[\cot \frac{x-s}{2} f(x)-\frac{1}{2} \cot \frac{x_{i}-s}{2}\right. \\
& +x^{2}
\end{aligned}
$$




$$
\begin{aligned}
& \cdot \sum_{i=0}^{n-1} \int_{x_{i}}^{x_{i+1}}\left[(x-s) \cot \frac{x-s}{2}-\frac{x_{i}-s}{2} \cot \frac{x_{i}-s}{2}\right. \\
& \left.-\frac{x_{i+1}-s}{2} \cot \frac{x_{i+1}-s}{2}\right] d x+\mathscr{R}_{n}(s)=-2 f(s) \\
& \cdot \pi \tan \frac{\tau \pi}{2}+\mathscr{R}_{n}(s),
\end{aligned}
$$

where

$$
\begin{aligned}
& \mathscr{R}_{n}(s)=R_{1}+R_{2}, \\
& R_{1}=\sum_{i=0, i \neq m}^{n-1} \int_{x_{i}}^{x_{i+1}}\left[\cot \frac{x-s}{2}-\frac{1}{2} \cot \frac{x_{i}-s}{2}-\frac{1}{2}\right. \\
& \left.\quad \cdot \cot \frac{x_{i+1}-s}{2}\right] \frac{1}{2} f^{\prime \prime}\left(\beta_{i 1}\right)(x-s)^{2} d x
\end{aligned}
$$

$$
\begin{aligned}
& -\sum_{i=0, i \neq m}^{n-1} \int_{x_{i}}^{x_{i+1}} \frac{f^{\prime \prime}\left(\alpha_{i 1}\right)}{2}\left(x_{i}-x\right)^{2} \cot \frac{x_{i}-s}{2} d x \\
& -\sum_{i=0, i \neq m}^{n-1} \int_{x_{i}}^{x_{i+1}} \frac{f^{\prime \prime}\left(\alpha_{i 2}\right)}{2}\left(x_{i+1}-x\right)^{2} \cot \frac{x_{i+1}-s}{2} d x \\
& -\sum_{i=0, i \neq m}^{n-1} \int_{x_{i}}^{x_{i+1}} \frac{f^{\prime \prime}\left(\beta_{i 2}\right)(x-s)}{2}\left[\left(x_{i}-x\right) \cot \frac{x_{i}-s}{2}\right.
\end{aligned}
$$$$
\left.+\left(x_{i+1}-x\right) \cot \frac{x_{i+1}-s}{2}\right] d x
$$$$
R_{2}=\int_{x_{m}}^{x_{m+1}} E_{m}(x) \cot \frac{x-s}{2} d x \text {. }
$$

Now we estimate $\mathscr{R}_{n}(s)$ term by term. For the first part of $R_{1}$, we have

$$
\begin{aligned}
& \left|\sum_{i=0, i \neq m}^{n-1} \int_{x_{i}}^{x_{i+1}}\left[\cot \frac{x-s}{2}-\frac{1}{2} \cot \frac{x_{i}-s}{2}-\frac{1}{2} \cot \frac{x_{i+1}-s}{2}\right] \frac{1}{2} f^{\prime \prime}\left(\beta_{i 1}\right)(x-s)^{2} d x\right| \\
& \quad=\left|\sum_{i=0, i \neq m}^{n-1} \int_{x_{i}}^{x_{i+1}} k_{s}(x) \frac{\left[\cot ((x-s) / 2)-(1 / 2) \cot \left(\left(x_{i}-s\right) / 2\right)-(1 / 2) \cot \left(\left(x_{i+1}-s\right) / 2\right)\right](1 / 2) f^{\prime \prime}\left(\beta_{i 1}\right)(x-s)^{3}}{x-s} d x\right| \\
& \quad \leq C \max \left\{\left|k_{s}(x)\right|\right\} h^{2}\left(\int_{c}^{x_{m}} \frac{1}{s-x} d x+\int_{x_{m+1}}^{c+2 \pi} \frac{1}{x-s} d x\right)=C \max \left\{\left|k_{s}(x)\right|\right\} h^{2} \ln \frac{(c+2 \pi-s)(s-c)}{\left(x_{m+1}-s\right)\left(s-x_{m}\right)} \\
& \quad \leq C \max \left\{\left|k_{s}(x)\right|\right\}(|\ln h|+|\ln \gamma(\tau)|) h^{2} .
\end{aligned}
$$

For the second part of $R_{1}$, there is no singularity and we have

$$
\begin{aligned}
& \left|\sum_{i=0, i \neq m}^{n-1} \int_{x_{i}}^{x_{i+1}} \frac{f^{\prime \prime}\left(\alpha_{i 1}\right)}{2}\left(x_{i}-x\right)^{2} \cot \frac{x_{i}-s}{2} d x\right| \\
& =\left|\sum_{i=0, i \neq m}^{n-1} \int_{x_{i}}^{x_{i+1}} \frac{k_{s}(x)\left(f^{\prime \prime}\left(\alpha_{i 1}\right) / 2\right)\left(x_{i}-x\right)^{2} \cot \left(\left(x_{i}-s\right) / 2\right)}{x-s} d x\right| \\
& \leq C \max \left\{\left|k_{s}(x)\right|\right\} h^{2}\left(\int_{c}^{x_{m}} \frac{1}{s-x} d x+\int_{x_{m+1}}^{c+2 \pi} \frac{1}{x-s} d x\right) \\
& =C \max \left\{\left|k_{s}(x)\right|\right\} h^{2} \ln \frac{(c+2 \pi-s)(s-c)}{\left(x_{m+1}-s\right)\left(s-x_{m}\right)} \\
& \leq C \max \left\{\left|k_{s}(x)\right|\right\}(|\ln h|+|\ln \gamma(\tau)|) h^{2} .
\end{aligned}
$$

The third and fourth parts of $R_{1}$ can be similarly obtained. For $R_{2}$, by Lemmas 5 and 6 , then we get

$$
\begin{aligned}
\left|\mathscr{R}_{n}(s)\right| & \leq\left|R_{1}\right|+\left|R_{2}\right| \\
& \leq C \max \left\{\left|k_{s}(x)\right|\right\}(|\ln h|+|\ln \gamma(\tau)|) h^{2}
\end{aligned}
$$

and the proof is completed.
Remark 7. By the identity in [27],

$$
\pi \cot \pi x=\sum_{l=-\infty}^{l=\infty} \frac{1}{x+l}
$$

then we get

$$
\begin{aligned}
& \cot \frac{x-s}{2}=\frac{2}{x-s}+\sum_{l=1}^{\infty} \frac{2}{x-s-2 l \pi}+\sum_{l=1}^{\infty} \frac{2}{x-s+2 l \pi}, \\
& \cot \frac{x-s}{2}-\cot \frac{\widehat{x}_{m}-s}{2} \\
& =\frac{2}{x-s}+\sum_{l=1}^{\infty} \frac{2}{x-s-2 l \pi}+\sum_{l=1}^{\infty} \frac{2}{x-s+2 l \pi} \\
& =\frac{2\left(\hat{x}_{m}-x\right)}{(x-s)\left(\hat{x}_{m}-s\right)}
\end{aligned}
$$


TABLE 1: An error estimate of the trapezoidal rule with $s=x_{[n / 4]}+(\tau+1) h / 2$.

\begin{tabular}{lcccc}
\hline & $\tau=0$ & $\tau=-\frac{2}{3}$ & $\tau=\frac{2}{3}$ & $\tau=\frac{1}{2}$ \\
\hline 8 & 0 & $-9.3692 e+000$ & $2.0089 e+000$ & $1.7335 e+000$ \\
16 & $-8.8818 e-016$ & $-1.0148 e+001$ & $6.8071 e+000$ & $4.1887 e+000$ \\
32 & $1.1546 e-014$ & $-1.0521 e+001$ & $8.9647 e+000$ & $5.2932 e+000$ \\
64 & $2.6645 e-015$ & $-1.0703 e+001$ & $9.9570 e+000$ & $5.8039 e+000$ \\
128 & $-4.2633 e-014$ & $-1.0793 e+001$ & $1.0429 e+001$ & $6.0477 e+000$ \\
256 & $-8.8818 e-015$ & $-1.0838 e+001$ & $1.0658 e+001$ & $6.1665 e+000$ \\
\hline
\end{tabular}

TABLE 2: An error estimate of the trapezoidal rule with $s=x_{0}+(\tau+1) h / 2$.

\begin{tabular}{ccccc}
\hline & $\tau=0$ & $\tau=-\frac{2}{3}$ & $\tau=\frac{2}{3}$ & $\tau=\frac{1}{2}$ \\
\hline 8 & $2.6645 e-015$ & $-1.2210 e+001$ & $1.5259 e+001$ & $8.7150 e+000$ \\
16 & $1.5099 e-014$ & $-1.1571 e+001$ & $1.3803 e+001$ & $7.8365 e+000$ \\
32 & $5.3291 e-015$ & $-1.1233 e+001$ & $1.2510 e+001$ & $7.1371 e+000$ \\
64 & $-3.0198 e-014$ & $-1.1059 e+001$ & $1.1736 e+001$ & $6.7284 e+000$ \\
128 & $2.4869 e-014$ & $-1.0971 e+001$ & $1.1319 e+001$ & $6.5102 e+000$ \\
256 & $-2.6645 e-015$ & $-1.0927 e+001$ & $1.1103 e+001$ & $6.3978 e+000$ \\
\hline
\end{tabular}

TABLE 3: An error estimate of the modified trapezoidal rule with $s=x_{[n / 4]}+(\tau+1) h / 2$.

\begin{tabular}{lcccr}
\hline & $\tau=0$ & $\tau=-\frac{2}{3}$ & $\tau=\frac{2}{3}$ & $\tau=\frac{1}{2}$ \\
\hline 8 & 0 & $3.5527 e-015$ & $8.8818 e-016$ & 0 \\
16 & $-8.8818 e-016$ & $-1.4211 e-014$ & $1.1768 e-014$ & $-5.3291 e-015$ \\
32 & $1.1546 e-014$ & $-2.1316 e-014$ & $1.5099 e-014$ & $-7.9936 e-015$ \\
64 & $2.6645 e-015$ & $8.1712 e-014$ & $-5.5511 e-014$ & $-4.2744 e-014$ \\
128 & $-4.2633 e-014$ & $6.3949 e-014$ & $-6.1284 e-014$ & $3.9413 e-014$ \\
256 & $-8.8818 e-015$ & $-2.9132 e-013$ & $2.4958 e-013$ & $5.4956 e-015$ \\
\hline
\end{tabular}

$$
\begin{aligned}
& +\sum_{l=1}^{\infty} \frac{2\left(\hat{x}_{m}-x\right)}{(x-s-2 l \pi)\left(\hat{x}_{m}-s-2 l \pi\right)} \\
& +\sum_{l=1}^{\infty} \frac{2\left(\hat{x}_{m}-x\right)}{(x-s+2 l \pi)\left(\hat{x}_{m}-s+2 l \pi\right)} .
\end{aligned}
$$

We can prove that the error expansion of this paper has the same error expansion of [25] for the Cauchy principal integral defined on the interval. Based on the error expansion, we get the same superconvergence point with local coordinate point equal to zero. rule

Based on Theorem 4, we present the modified trapezoidal

$$
\widetilde{I}_{n}(f ; s)=I_{n}(f ; s)-2 f(s) \pi \tan \frac{\tau \pi}{2} .
$$

\section{Numerical Example}

In this section, computational results are reported to confirm our theoretical analysis.

Example. We consider the singular integral with $f(x)=$ $2 \cos x+\sin x c=0 . s=x_{[n / 4]}+(\tau+1) h / 2$ and $s=$ $x_{0}+(\tau+1) h / 2$ with $\tau=0$ are the superconvergence point.

From Tables 1 and 2, we know that the superconvergence point is 0 with the coordinate location of singular point equal to zero, while, for the local coordinate of singular point not equal to zero, it is not convergence in general which coincides with our analysis.

For the modified classical trapezoidal rule, from Tables 3 and 4 , for the nonsuperconvergence point and the superconvergence point, we all get the superconvergence phenomenon. 
TABLE 4: An error estimate of the modified trapezoidal rule with $s=x_{0}+(\tau+1) h / 2$.

\begin{tabular}{lcccc}
\hline & $\tau=0$ & $\tau=-\frac{2}{3}$ & $\tau=\frac{2}{3}$ & $\tau=\frac{1}{2}$ \\
\hline 8 & $2.6645 e-015$ & $-7.9936 e-015$ & $2.1316 e-014$ & $-1.2434 e-014$ \\
16 & $1.5099 e-014$ & $4.7962 e-014$ & $3.1974 e-014$ & $-8.8818 e-015$ \\
32 & $5.3291 e-015$ & $1.2079 e-013$ & $-6.7502 e-014$ & $-4.6185 e-014$ \\
64 & $-3.0198 e-014$ & $3.2863 e-013$ & $-7.4607 e-014$ & $6.3949 e-014$ \\
128 & $2.4869 e-014$ & $2.4780 e-013$ & $2.4158 e-013$ & $3.1974 e-014$ \\
256 & $-2.6645 e-015$ & $8.0824 e-013$ & $-4.5830 e-013$ & $4.1744 e-013$ \\
\hline
\end{tabular}

\section{Conflict of Interests}

The author declares that there is no conflict of interests regarding the publication of this paper.

\section{Acknowledgments}

The work of Jin Li was supported by the National Natural Science Foundation of China (Grant no. 11471195, Grant no. 11101247, and Grant no. 11201209) and China Postdoctoral Science Foundation (Grant nos. 2013M540541 and 2015T80703).

\section{References}

[1] D. H. Yu, Natural Boundary Integral Method and Its Applications, Kluwer Academic Publishers, 2002.

[2] G. Criscuolo and G. Mastroianni, "On the uniform convergence of Gaussian quadrature rules for Cauchy principal value integrals," Numerische Mathematik, vol. 54, no. 4, pp. 445-461, 1989.

[3] G. Criscuolo and G. Mastroianni, "Gaussian formulas for computing Cauchy principal value integrals, and their convergence," Calcolo, vol. 22, no. 3, pp. 391-411, 1985.

[4] K. Diethelm, "Gaussian quadrature formulae of the third kind for Cauchy principal value integrals: basic properties and error estimates," Journal of Computational and Applied Mathematics, vol. 65, no. 1-3, pp. 97-114, 1995.

[5] K. Diethelm, "Asymptotically sharp error bounds for a quadrature rule for Cauchy principal value integrals based on piecewise linear interpolation," Approximation Theory and Its Applications, vol. 11, no. 4, pp. 78-89, 1995.

[6] N. I. Ioakimidis, "On the uniform convergence of Gaussian quadrature rules for Cauchy principal value integrals and their derivatives," Mathematics of Computation, vol. 44, no. 169, pp. 191-198, 1985.

[7] T. Hasegawa, "Uniform approximations to finite Hilbert transform and its derivative," Journal of Computational and Applied Mathematics, vol. 163, no. 1, pp. 127-138, 2004.

[8] G. Monegato, "Convergence of product formulas for the numerical evaluation of certain two-dimensional Cauchy principal value integrals," Numerische Mathematik, vol. 43, no. 2, pp. 161$173,1984$.

[9] S. Amari, "Evaluation of Cauchy principal-value integrals using modified Simpson rules," Applied Mathematics Letters, vol. 7, no. 3, pp. 19-23, 1994.

[10] K. Diethelm, "Modified compound quadrature rules for strongly singular integrals," Computing, vol. 52, no. 4, pp. 337354, 1994.
[11] P. Köhler, "On the error of quadrature formulae for Cauchy principal value integrals based on piecewise interpolation," Approximation Theory and Its Applications, vol. 13, no. 3, pp. 5869, 1997.

[12] J. Li and D. H. Yu, "The superconvergence of certain twodimensional Cauchy principal value integrals," CMES, vol. 71, no. 4, pp. 331-346, 2011.

[13] J. Li and D. H. Yu, "The superconvergence of certain twodimensional Hilbert singular integrals," Computer Modeling in Engineering \& Sciences, vol. 82, no. 3-4, pp. 233-252, 2011.

[14] C. Dagnino and E. Santi, "On the evaluation of one-dimensional Cauchy principal value integrals by rules based on cubic spline interpolation," Computing, vol. 43, no. 3, pp. 267-276, 1990.

[15] A. P. Orsi, "Spline approximation for Cauchy principal value integrals," Journal of Computational and Applied Mathematics, vol. 30, no. 2, pp. 191-201, 1990.

[16] G. Behforooz, "Approximation of Cauchy principal value integrals by piecewise Hermite quartic polynomials by spline," Applied Mathematics Letters, vol. 5, no. 1, pp. 75-78, 1992.

[17] P. Kim and U. J. Choi, "A quadrature rule of interpolatory type for Cauchy integrals," Journal of Computational and Applied Mathematics, vol. 126, no. 1-2, pp. 207-220, 2000.

[18] R. Kress, I. H. Sloan, and F. Stenger, "A sinc quadrature method for the double-layer integral equation in planar domains with corners," Journal of Integral Equations \& Applications, vol. 10, no. 3, pp. 291-317, 1998.

[19] P. Junghanns and B. Silbermann, "Numerical analysis for onedimensional Cauchy singular integral equations," Journal of Computational and Applied Mathematics, vol. 125, no. 1-2, pp. 395-421, 2000.

[20] A. Natarajan and N. Mohankumar, "A comparison of some quadrature methods for approximating Cauchy principal value integrals," Journal of Computational Physics, vol. 116, no. 2, pp. 365-368, 1995.

[21] F. Stenger, "Sinc approximation of Cauchy-type integrals over arcs," in Proceedings of the Meeting in Honor of David Elliott's 65th Birthday, 1998.

[22] C. F. Xu and Y. F. Yao, "Numerical calculation of Cauchy principal value integrals using wavelet functions," Numerical Mathematics. A Journal of Chinese Universities, vol. 20, no. 2, pp. 97-105, 1998.

[23] D. J. Liu, J. M. Wu, and D. H. Yu, "The superconvergence of the Newton-Cotes rule for Cauchy principal value integrals," Journal of Computational and Applied Mathematics, vol. 235, no. 3, pp. 696-707, 2010. 
[24] J. M. Wu and W. W. Sun, "The superconvergence of NewtonCotes rules for the Hadamard finite-part integral on an interval," Numerische Mathematik, vol. 109, no. 1, pp. 143-165, 2008.

[25] J. Li and D. H. Yu, "Error expansion of classical trapezoidal rule for computing Cauchy principal value integral," Computer Modeling in Engineering \& Sciences, vol. 93, no. 1, pp. 47-67, 2013.

[26] J. Li, J. E. Yang, and D. H. Yu, "Error expansion of classical mid-point rectangle rule for computing Cauchy principal value integrals on an interval," International Journal of Computer Mathematics, vol. 91, no. 10, pp. 2294-2306, 2014.

[27] L. C. Andrews, Special Functions of Mathematics for Engineers, McGraw-Hill, 2nd edition, 1992. 


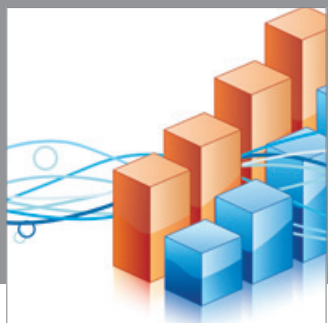

Advances in

Operations Research

mansans

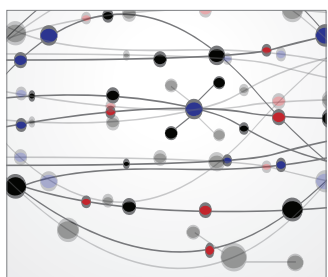

The Scientific World Journal
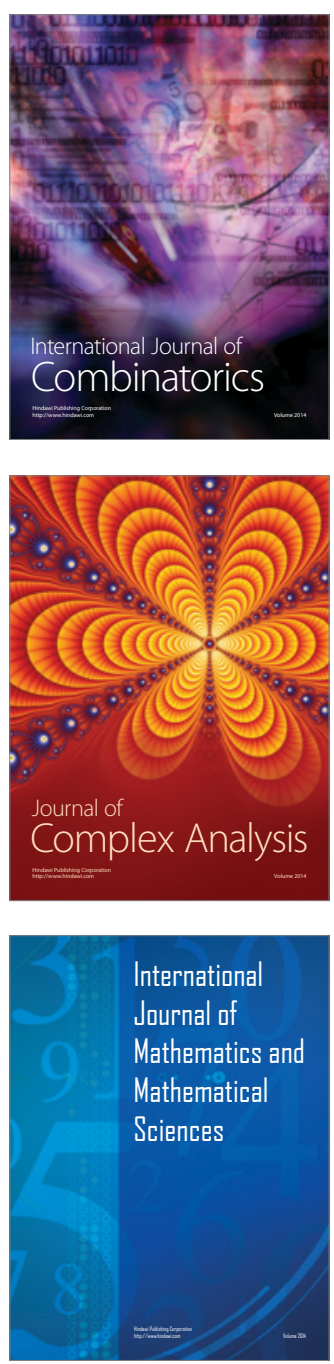
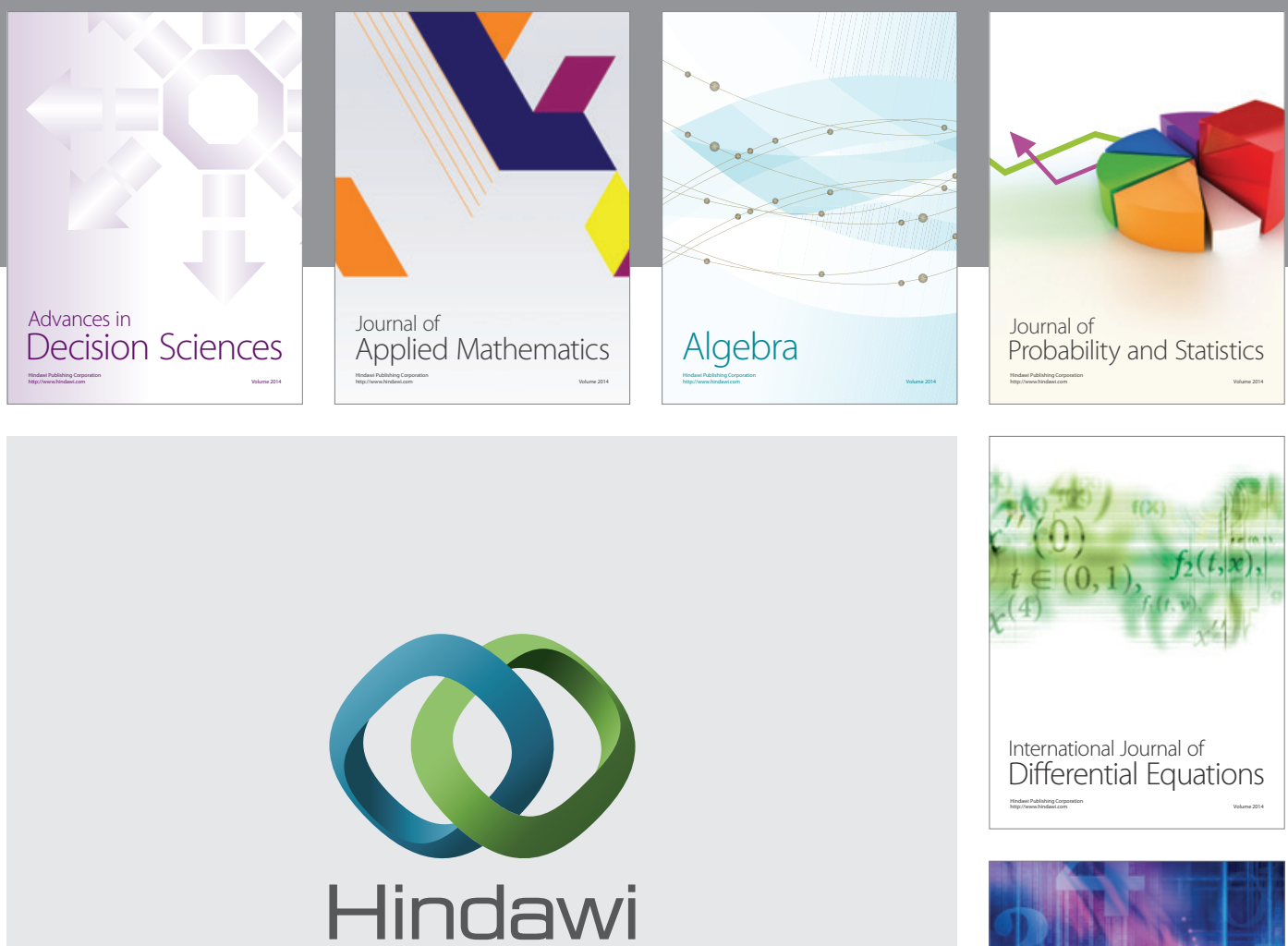

Submit your manuscripts at http://www.hindawi.com
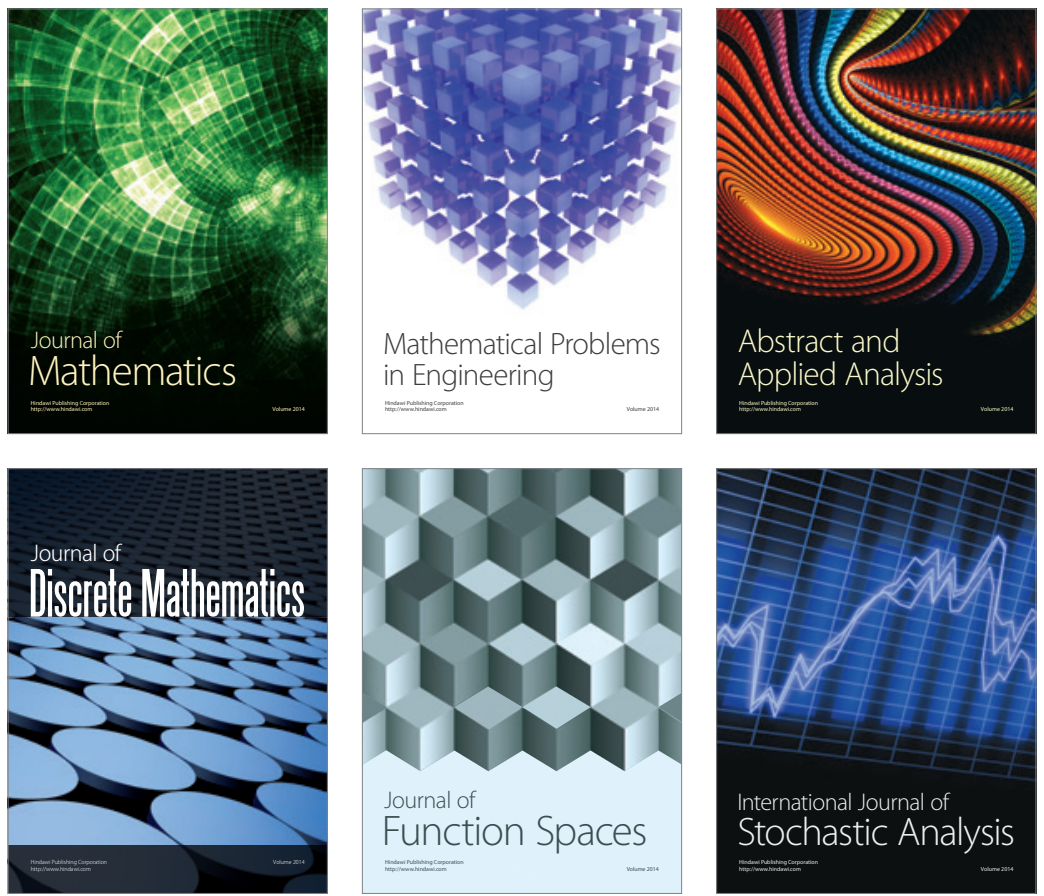

Journal of

Function Spaces

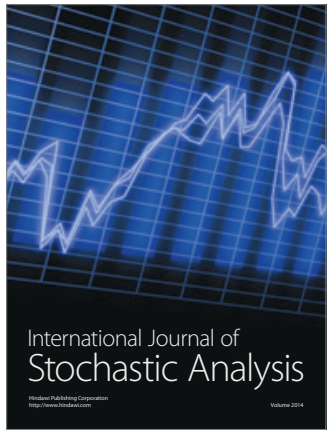

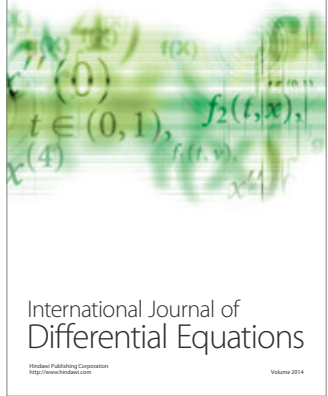
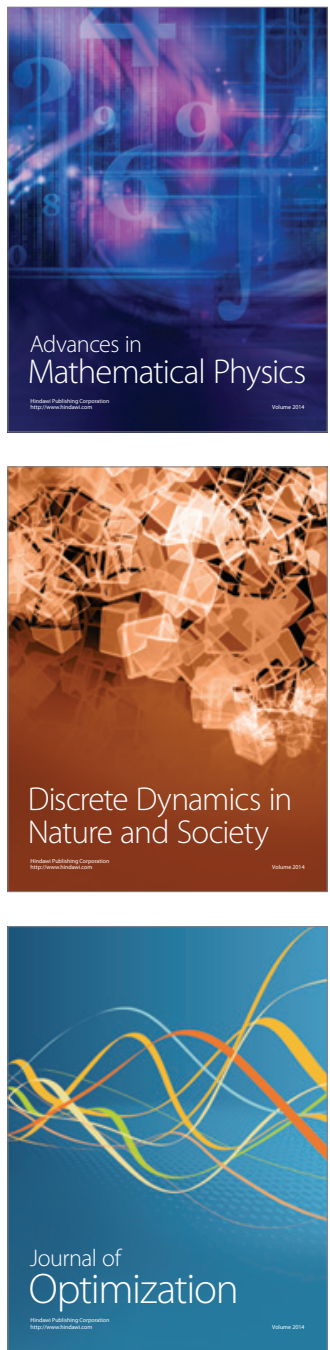